\title{
A Bayesian Analysis of the Molnupiravir Trial Data
}

\author{
Eric-Jan Wagenmakers \& Quentin F. Gronau
}

Affiliation: Psychological Methods Unit, University of Amsterdam

Email of corresponding author: EJ.Wagenmakers@gmail.com

Word count: 557

Address for correspondence:

Eric-Jan Wagenmakers

Department of Psychological Methods, room G 0.29

University of Amsterdam, Nieuwe Achtergracht 129B

Letter: PO Box 15906, 1001 NK Amsterdam

Parcel: Valckenierstraat 59, 1018 XE Amsterdam

On October 1st 2021, Merck issued a press release ${ }^{1}$ stating that: "molnupiravir (MK-4482, EIDD-2801), an investigational oral antiviral medicine, significantly reduced the risk of hospitalization or death at a planned interim analysis of the Phase 3 MOVe-OUT trial in at risk, non-hospitalized adult patients with mild-to-moderate COVID-19. At the interim analysis, molnupiravir reduced the risk of hospitalization or death by approximately $50 \% ; 7.3 \%$ of patients who received molnupiravir were either hospitalized or died through Day 29 following randomization (28/385), compared with $14.1 \%$ of placebo-treated patients $(53 / 377) ; \mathrm{p}=0.0012$. (...) Merck plans to submit an application for Emergency Use Authorization (EUA) to the U.S. FDA as soon as possible based on these findings and plans to submit marketing applications to other regulatory bodies worldwide." 
In order to quantify the evidence that molnupiravir is indeed effective, we conduct a Bayesian logistic regression with group membership as the predictor variable. ${ }^{2,3}$ Under the no-effect model $\mathrm{H}_{0}$, the $\log$ odds ratio equals $\psi=0$, whereas under the positive-effect model $\mathrm{H}_{+}, \psi$ is assigned a positive-only normal prior $\mathrm{N}_{+}(\mu, \sigma)$, reflecting the fact that the hypothesis of interest (i.e., molnupiravir is helpful, not harmful) is directional. A default analysis (i.e., $\mu=0, \sigma=1$ ) reveals very strong evidence for $\mathrm{H}_{+}{ }^{5}$ Specifically, the observed data are about 36 times more likely under $\mathrm{H}_{+}$than under $\mathrm{H}_{0}$. This evidence would increase the probability for $\mathrm{H}_{+}$from 0.50 before having seen the data to about 0.97 after having seen the data. Figure 1 shows that the evidence for $\mathrm{H}_{+}$is strong for a wide range of prior distributions based on combinations of $\mu$ in $[0,1.5]$ and $\sigma$ in $[.25,1]$.

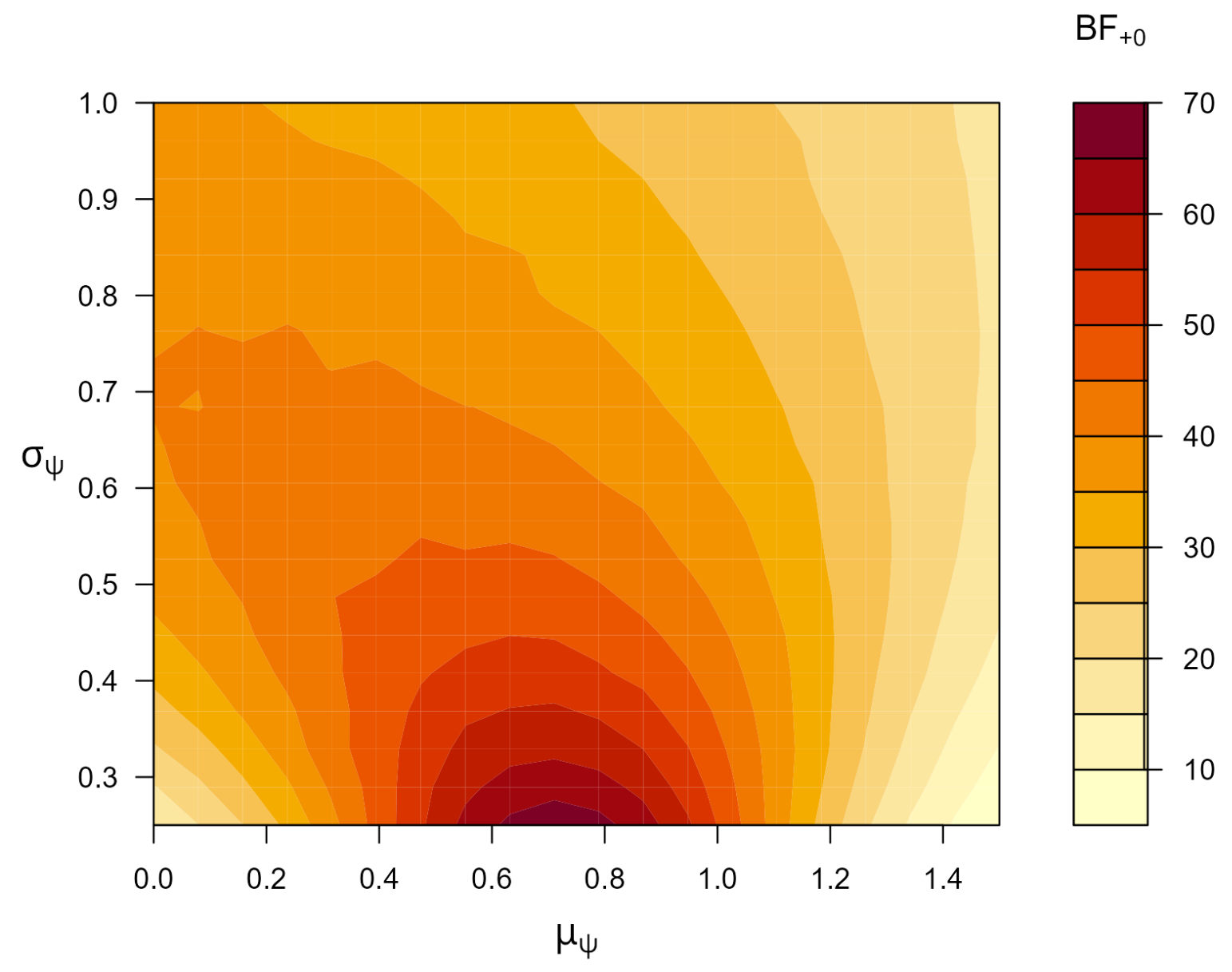

Figure 1. Across a wide range of prior distributions for the log odds ratio under $\mathrm{H}_{+}$, there is considerable evidence for the positive-effect model $H_{+}$over the no-effect model $H_{0}$. Figure from JASP (jasp-stats.org).

Disregarding $\mathrm{H}_{0}$ for the purpose of parameter estimation, Figure 2 shows the prior and posterior distribution for the log odds ratio under a nondirectional alternative hypothesis. Although there 
remains considerable uncertainty about the exact size of the effect, most posterior mass is on values that would be considered to be clinically meaningful.

$$
\text { median }=0.682
$$

95\% Cl: $[0.216,1.147]$

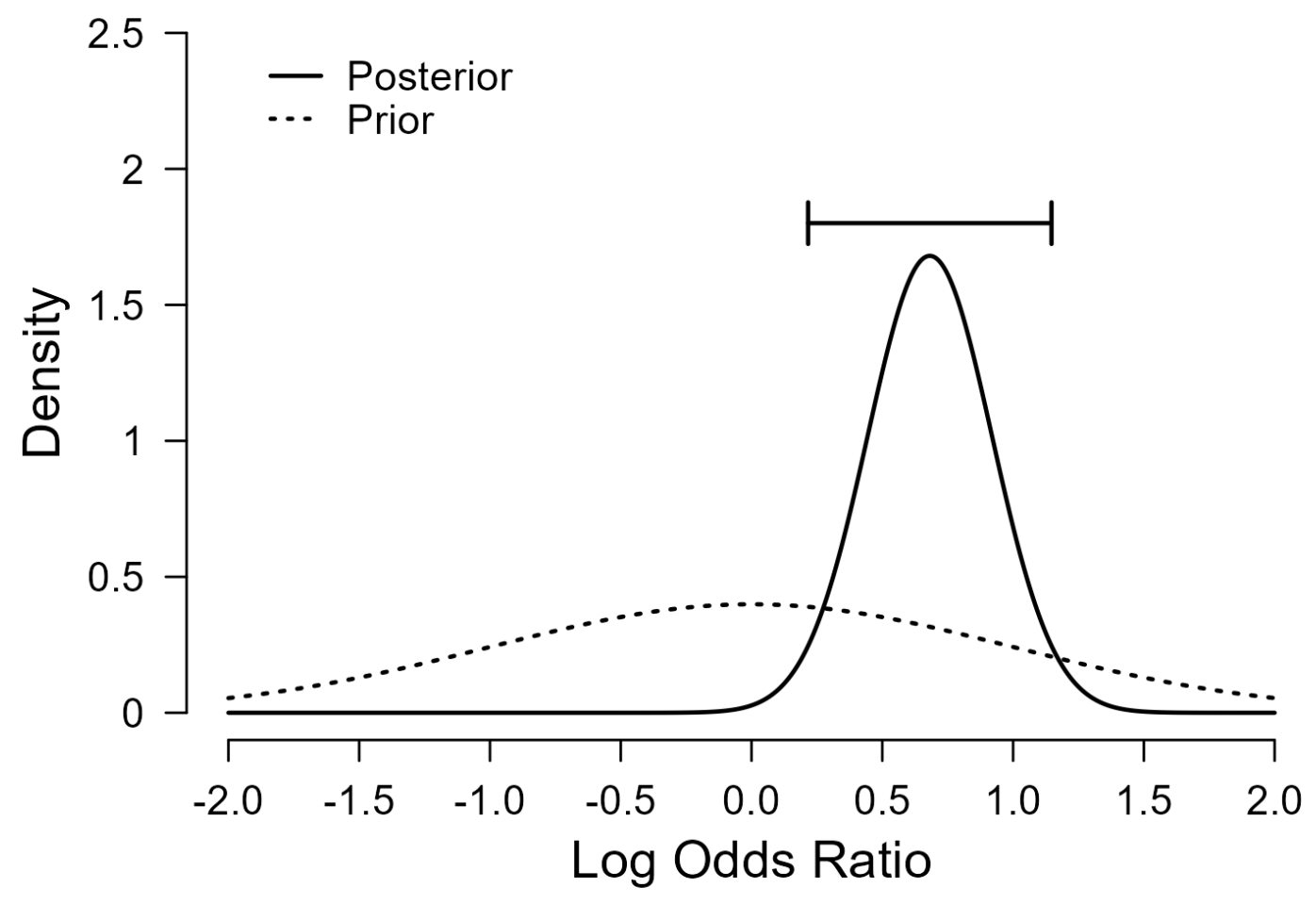

Figure 2. Prior and posterior distribution of the log odds ratio $\psi$ for the effect of molnupiravir. Under a nondirectional standard normal prior distribution, it is 95\% probable that the true value of $\psi$ falls between 0.216 and 1.147. Figure from JASP (jasp-stats.org).

The same information is presented in Figure 3, but now on the probability scale. Note the separation between the posterior distributions for the two groups. 


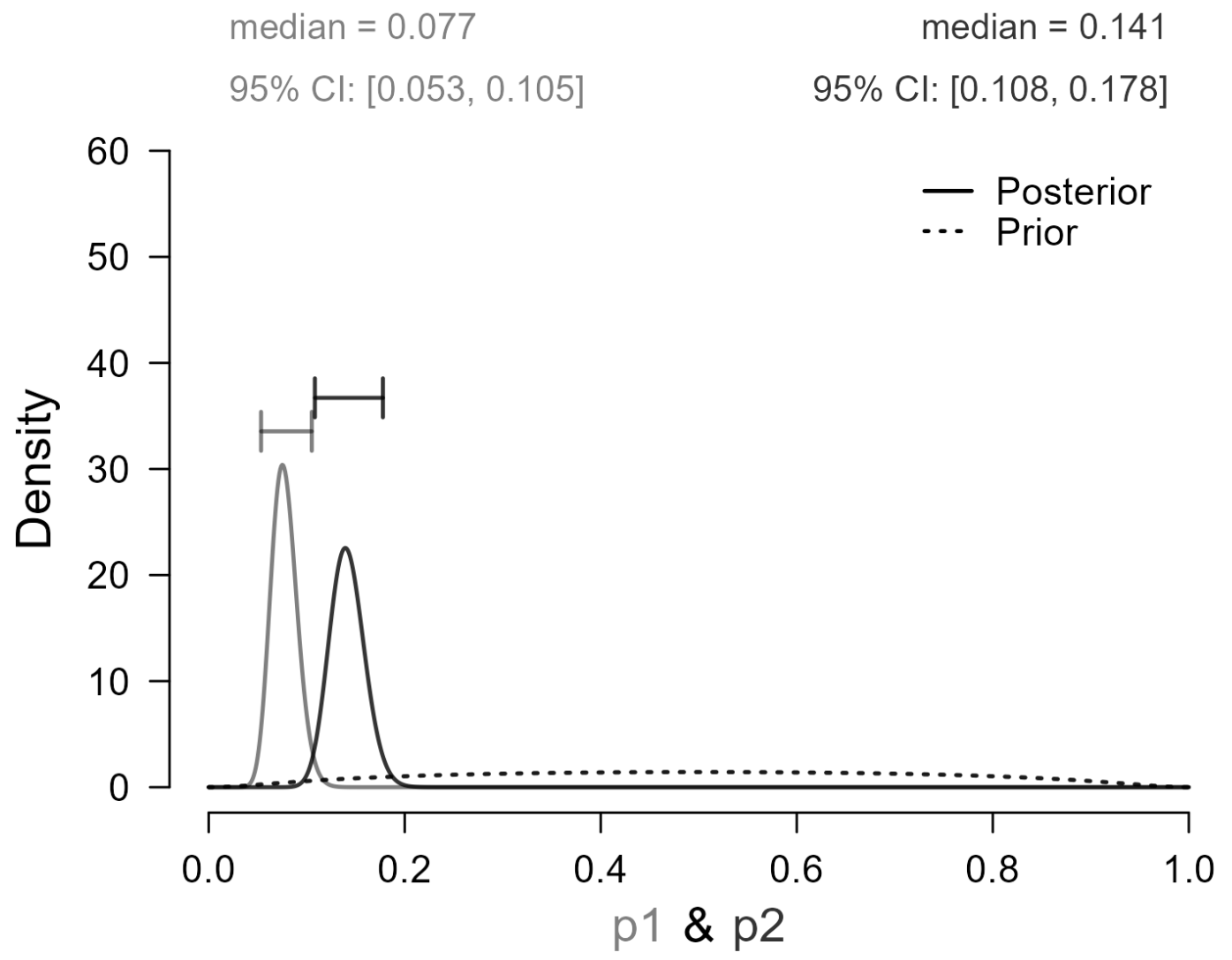

Figure 3. The difference between the two posterior distributions indicates the size of the effect on the probability scale. The gray " $p 1$ " and the black " 2 " indicate the probability of hospitalization or death in the molnupiravir group and the placebo group, respectively. Figure from JASP (jasp-stats.org).

In sum, our Bayesian analysis confirms that the molnupiravir data are indeed promising. A default analysis raises the probability for $\mathrm{H}_{+}$from 0.50 to about 0.97 and is robust to a range of prior specifications. Nevertheless, the evidence is not so compelling as to rule out $\mathrm{H}_{0}$ almost entirely - its strength is the statistical equivalent of throwing two consecutive sixes with a fair die.

\section{References}

1. Merck and Ridgeback's Investigational Oral Antiviral Molnupiravir Reduced the Risk of Hospitalization or Death by Approximately 50 Percent Compared to Placebo for Patients with Mild or Moderate COVID-19 in Positive Interim Analysis of Phase 3 Study. Oct 2021. 
https://www.merck.com/news/merck-and-ridgebacks-investigational-oral-antiviral-molnu piravir-reduced-the-risk-of-hospitalization-or-death-by-approximately-50-percent-compar ed-to-placebo-for-patients-with-mild-or-moderat.

2. Kass RE, Vaidyanathan SK. Approximate Bayes factors and orthogonal parameters, with application to testing equality of two binomial proportions. Journal of the Royal Statistical Society: Series B (Methodological) 1992;54:129-44.

3. Gronau QF, Raj K. N. A., Wagenmakers EJ. (in press). Informed Bayesian inference for the A/B test. Journal of Statistical Software.

4. Jeffreys, H. Theory of Probability. 1st ed. Oxford University Press, Oxford, UK, 1939. 